\title{
THE MILLIPEDE GENUS NEDYOPUS ATTEMS, 1914 (DIPLOPODA: POLYDESMIDA: PARADOXOSOMATIDAE) FROM VIETNAM
}

\author{
Nguyen Duc Anh \\ Institute of Ecology and Biological Resources, VAST, ducanh@iebr.ac.vn
}

\begin{abstract}
The genus Nedyopus Attems, 1914 consisting of 22 known species and subspecies is distributed mainly in East Asia (Japan, Taiwan) and expansively occurs in Vietnam (three species). The paper presents taxonomy and diagnosis of three Nedyopus species recorded in Vietnam: N. dawydoffiae (Attems, 1953), N. mahunkai (Korsós \& Golovatch, 1989) and N. pictus (Brölemann, 1916). Of those, $N$. mahunkai has been widely known in northeastern Vietnam, and $N$. dawydoffiae has been found in southern Vietnam. The species N. pictus has been recorded only in Tam Dao National Park. A key to species is also provided.
\end{abstract}

Keywords: Polydesmida, Paradoxosomatidae, Nedyopus, Millipede, Vietnam

\section{INTRODUCTION}

The millipede genus Nedyopus was established for the species Orthomorpha cingulata Attems, 1898 by Attems (1914) [1]. Three years later, Brölemann (1916) [4] proposed a new monotypic genus Vaulogerodesmus with a single species $V$. pictus. This genus was later synonymized with the genus Nedyopus by Attems (1937) [2]. Since then, numerous species have been described under genera Nesodyopus and Varyomorpha [11, 13, 14], but their generic positions were still doubtful [11].

Recently, Chen et al. (2006) [5] revised the tribe Nedyopodini consisting of only the genus Nedyopus Attems, 1914. They synonymized all genera Vaulogerodesmus Brölemann, 1916, Nesodyopus Verhoeff, 1940 and Varyomorpha Wang, 1957 with the genus Nedyopus. Consequently, the number of species has increased to 13 species plus 9 subspecies. Almost all species have been recorded in Taiwan, Japan and Korea whereas some occur in southern China, Vietnam and Indonesia. Only three species have been found in Vietnam [6].

The paper presents taxonomy and distribution of the genus Nedyopus recorded in Vietnam, and provides a key to species.

Checklist and distribution of Nedyopus species:

Nedyopus cingulatus (Attems, 1898), from Japan;
Nedyopus beroni (Golovatch, 1995), from Southern China;

Nedyopus boninensis Verhoeff, 1940, from Bonin Islands, Japan;

Nedyopus caudatus Chen, Golovatch \& Chang, 2006, from Taiwan;

Nedyopus dawydoffiae (Attems, 1953), from Vietnam (Lam Dong);

Nedyopus hiburizimensis hiburizimensis Miyosi, 1955 from Japan;

Nedyopus hiburizimensis aquilus Haga, 1968 from Japan;

Nedyopus hsientienensis (Wang, 1957), from Taiwan;

Nedyopus koreanus koreanus Verhoeff, 1936, from Korea;

Nedyopus koreanus niponianus Verhoeff, 1940, from Japan;

Nedyopus latus Chen, Golovatch \& Chang, 2006, from Taiwan;

Nedyopus mahunkai (Korsós \& Golovatch, 1989), from Vietnam;

Nedyopus patrioticus (Attems, 1898), from Japan, Korea;

Nedyopus patrioticus var. unicolor (Carl, 1902), from Indonesia (Sumatra);

Taiwan;

Nedyopus pectinatus (Wang, 1957), from

Nedyopus pictus (Brölemann, 1916), from Vietnam; 
Nedyopus picturatus (Golovatch, 1995), from Southern China;

Nedyopus tambanus tambanus (Attems, 1901), from Center Japan (Tamba, Kuwadagori, Hozuyama);

Nedyopus tambanus ikaonus (Attems, 1909), from Japan (Ikao);

Nedyopus tambanus mangaesinus (Attems, 1909), from Japan (Mangaesi);

Nedyopus venustus Shinohara, 1963, from Japan;

Nedyopus wui Chen, Golovatch \& Chang, 2006, from Taiwan;

\section{MATERIALS AND METHODS}

All fresh materials was collected during field trips in Vietnam, and preserved in ethanol $75 \%$. All were examined using a microscope Olympus SZX10 with a drawing tube attached. SEM images were made using a machine ABT 32 (Topcon Technohouse Co. Ltd) in the Faculty of Science, Ibaraki University, Japan.

All specimens are kept in Department of Soil Environmental Ecology, Institute of Ecology and Biological Resources (IEBR), Hanoi, Vietnam.

\section{RESULTS AND DISCUSSION}

\section{Systematics}

\section{Nedyopus Attems, 1914}

Nedyopus Attems, 1914: 200 [1]; Attems 1937: 137 [2]; Verhoeff, 1940: 141 [13]; Jeekel 1968: 74 75 [11]; Chen et al. 2006: 4001-4002 [5].

Vaulogerodesmus Brölemann, 1916 [4], synonymized by Attems 1937: 137 [2]; but revalidated by Jeekel 1968: 61 [11]; and then re-synonymized with Nedyopus by Chen et al. 2006: 4001 [5];
Nesodyopus Verhoeff, 1940: 141 [13], proposed as subgenus of Nedyopus; erected to full genus by Jeekel 1968: 75 [11]; then resynonymized by Chen et al. 2006: 4001 [5].

Varyomorpha Wang, 1957: 104 [14], proposed as a subgenus of Oxidus, erected to full genus by Jeekel 1968: 77 [11], with quotation "uncertae sedis"; then synonymized by Chen et al. 2006: 4001 [5].

Type species: Orthomorpha cingulata Attems, 1898, by original designation.

Diagnosis: Paradoxosomatid species with medium size (ca. 30-40 mm long and 4.0-5.0 $\mathrm{mm}$ wide). Paraterga modestly - to welldeveloped. Pleurosternal carinae present, usually on body rings 2-7. Metaterga usually smooth, without any traces of setae. Sternal process between coxae 4 always present.

Gonopod coxae relatively long, slightly shorter than femorite, with a ventrodistal part sparsely setosa. Prefemorite densely setosa, set off from femorite by a distinctly oblique suture laterally. Femorite elongate and usually expanded distally, strongly twisted at base, so that prostatic groove running entirely on lateral side of femorite. The demarcation between femorite and postfemoral region missing. Solenomere flagelliform, partly sheathed by solenophore consisting of both well-developed lamina medialis and lamina lateralis.

Remarks: The taxonomic history of the genus Nedyopus is rather complicated, and was reviewed recently by Chen et al. (2006) [5]. The genus is considered to occur in East Asia (Taiwan, Korea and Japan) and to expand to Vietnam and Indonesia (Sumatra). It currently comprises 13 species and nine subspecies, of which only three species occur on Vietnam (Enghoff et al. 2004) [6] and one in Indonesia (Sumatra Island).

\section{Key to species of the genus Nedyopus from Vietnam}

1. Body larger, about $40 \mathrm{~mm}$ long. The fifth sternum with a large laminiform process between male coxae 4 (Figure 1B). Gonopod femorite parallel-sided, slightly broadened distally (Figures 1C-D, 2) .N. dawydoffiae

Body smaller, about $32 \mathrm{~mm}$ long. The fifth sternum with two rounded cones between male coxae 4. Gonopod femorite broadened distally, not parallel-sided. 
2. Paraterga modestly developed, always angular caudally, not protruding beyond posterior tergal contour (Figure 5A). The fifth sternum with two independent, setiferous conial processes between coxae 4 (Figure 5B). Gonopod solenophore with characteristically subsecuriform lamina medialis (Figures 5C-D, 6). N. pictus

- $\quad$ Paraterga well developed, nearly pointed caudally, sometimes exceeding over posterior tergal contour (Figure 3A). The fifth sternum with two closed, setiferous conial processes between coxae 4 (Figure 3B). Gonopod solenophore without subsecuriform lamina medialis (Figures 3C-D, 4)..... N. mahunkai

\section{Nedyopus dawydoffiae (Attems, 1953) (Figs 1-2)}

Helicorthomorpha dawydoffiae Attems, 1953: 169-170, figures 57-58 [3]; Jeekel 1968: 64, "unnamed genus" [11].

Vaulogerodesmus dawydoffiae: - Golovatch, 1983: 182 [8]; Golovatch et al. 2002: 262, figures 15-17 [10]; Enghoff et al. 2004: 40 [6].

Nedyopus dawydoffiae: - Chen et al. 2006: 4001 [5]; Golovatch 2009: 4, figures 14-22 [9].

Diagnosis: The species can be distinguished from its congeners by bigger body size (about $40 \mathrm{~mm}$ long and $5 \mathrm{~mm}$ wide); paraterga modestly developed, rounded, not surpassing posterior contour of metaterga; the fifth sternum with a large lamina between coxae 4; gonopod femorite more or less parallel-sided, slightly broadened distally.

Previous records: Lam Dong; Dong Nai (Cat Tien National Park (=NP)) - (Attems 1953; Golovatch 2009; Golovatch \& Semenyuk 2010) $[3,7,9]$.

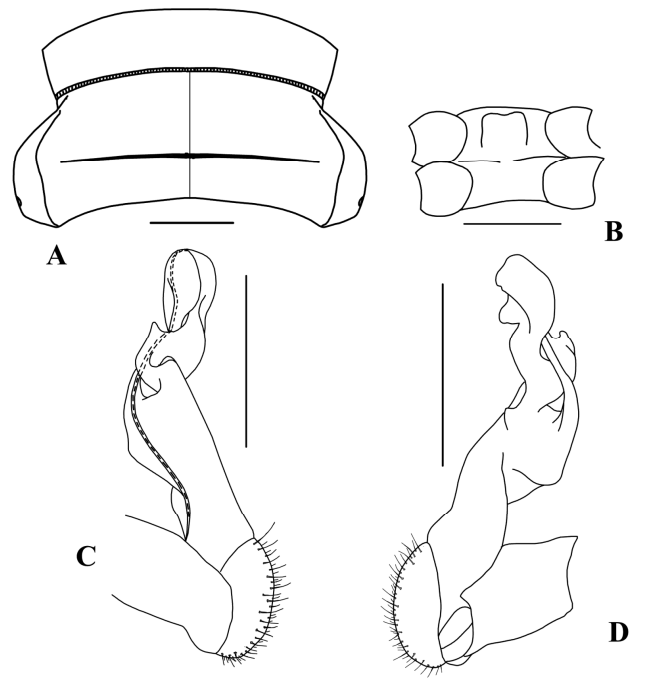

Figure 1. Nedyopus dawydoffiae (Attems, 1953), from Bi Doup National Park

A. Body ring 10, dorsal view; B. sternum 5th, ventral view; C-D. left gonopod, lateral view and mesal view, respectively. Scale bar $=1 \mathrm{~mm}$.

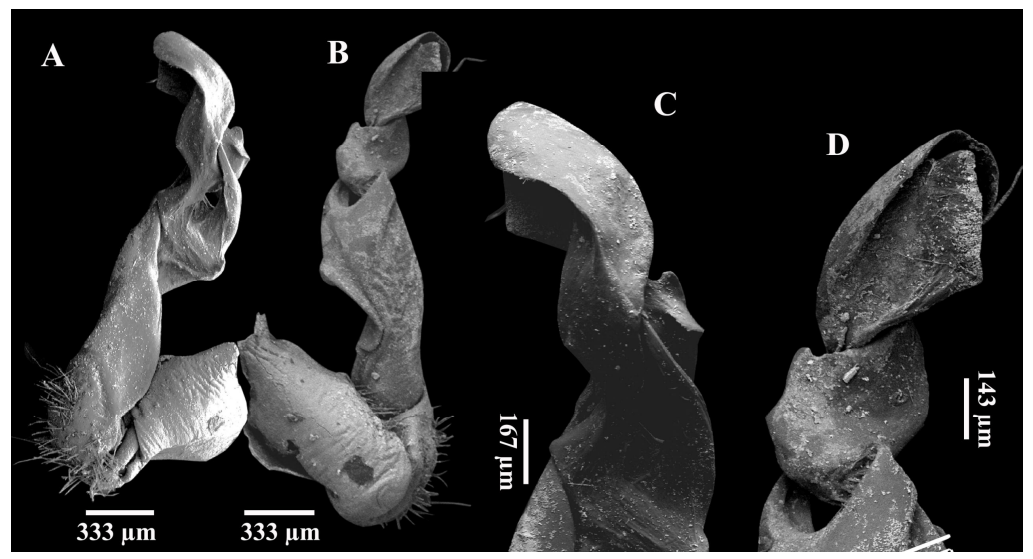

Figure 2. Nedyopus dawydoffiae (Attems, 1953), from Bi Doup National Park Left gonopod, mesal view (A), lateral view (B); postfemoral region, mesal view (C), lateral view (D) 
Material examined: Lam Dong: 5 males, 5 females (IEBR-6) Bi Doup-Nui Ba NP, primary forest, 1,400-1,500m a.s.l., 23 March-23 April 2008, Anh D. Nguyen; 1 male (IEBR-7) same locality, but 1,500-1,800 m a.s.l., 20 April-9 May 2009; Dong Nai: 1 female (IEBR-84) Cat Tien NP, forest, on tree trunk, 26-27 July 2009, Anh D. Nguyen; Dak Lak: 1 male (IEBR-250) Chu Yan Sin NP, forest, 1,000-1,500 m a.s.l., 28 March- 12 April 2012, Le Son.

Distribution: Only known from southern Vietnam (Fig. 7).

\section{Nedyopus mahunkai (Korsós \& Golovatch, 1989) (Figs 3-4)}

Vaulogerodesmus mahunkai Korsós \& Golovatch, 1989: 217-219, figures 11-14 [12]; Golovatch et al. 2002: 263, figures 18-21 [10]; Enghoff et al. 2004: 37 [6].

Nedyopus mahunkai:- Chen et al. 2006: 4001 [5].

Diagnosis: The species can be distinguished from its congeners by smaller body size (less than $40 \mathrm{~mm}$ long and $5 \mathrm{~mm}$ wide); paraterga well developed, sometimes surpassing posterior contour of metaterga; the fifth sternum with two closed conial processes or a strongly bifid process between coxae 4; gonopod femorite not parallel-sided, strongly broadened distally; solenophore without characteristically subsecuriform lamina medialis.

Previous records: Vinh Phuc (Tam Dao NP), Ninh Binh (Cuc Phuong NP) - (Korsós \& Golovatch 1989) [12].

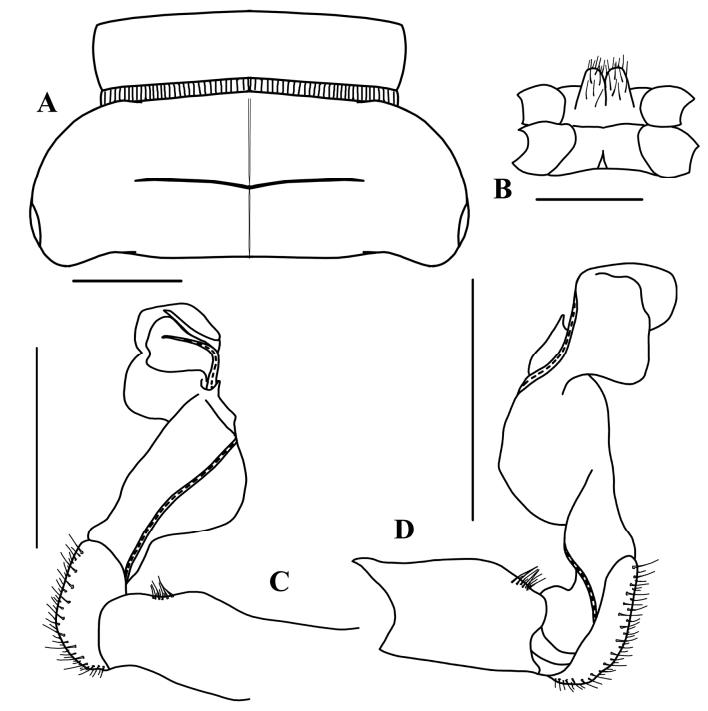

Figure 3. Nedyopus mahunkai (Korsós \& Golovatch, 1989), from Tam Dao National Park

A: Body ring 10, dorsal view; B: sternum 5th, ventral view; $C$-D: right gonopod, lateral view and mesal view, respectively. Scale bar $=1 \mathrm{~mm}$.

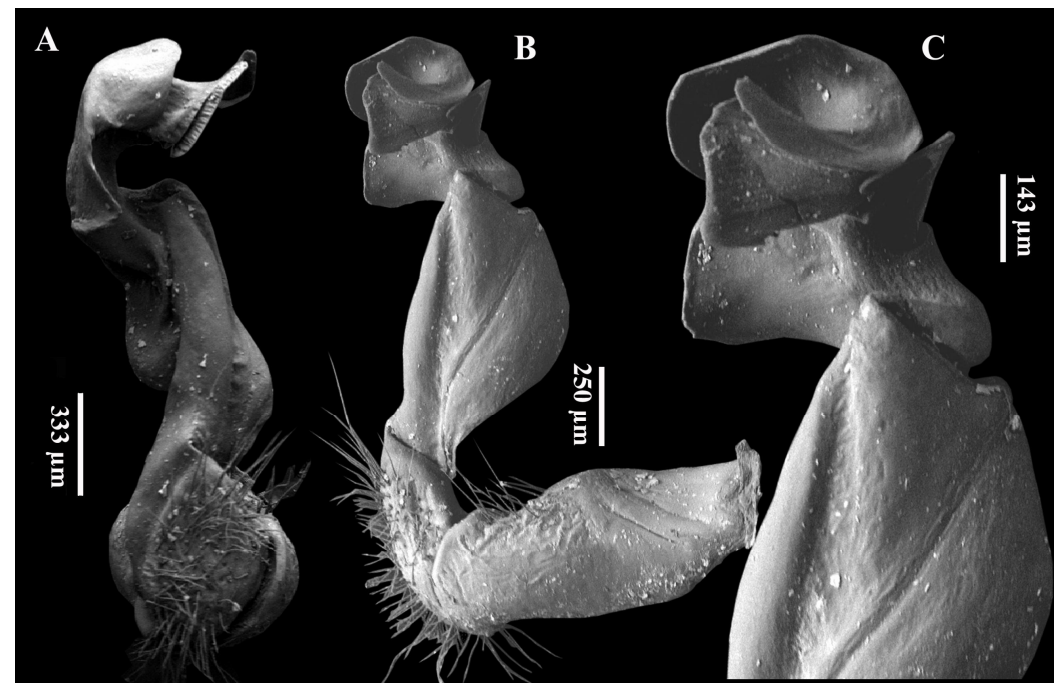

Figure 4. Nedyopus mahunkai (Korsós \& Golovatch, 1989), from Tam Dao National Park Right gonopod, ventral view (A), lateral view (B); postfemoral region, lateral view (C) 
Material examined: Phu Tho: 1 male, 1 female, 1 juvenile (IEBR-23) Xuan Son NP, primary forest, 15 January 2006, Nguyen Van Quang; Vinh Phuc: 1 male (IEBR-24) Tam Dao $\mathrm{NP}$, garden around the town, $900 \mathrm{~m}$ a.s.l., 1 October 2005, Anh D. Nguyen; 1 male, 1 female (IEBR-81) same locality as sample IEBR-24, forest, on the way to Rung Rinh peak, 900-1,100 m a.s.l., 15-18 October 2010, Anh D. Nguyen; 1 female (IEBR-82) same locality as sample IEBR-24, 15-18 October 2010, Anh D. Nguyen; 2 females (IEBR-170), 2 males, 2 females (IEBR-171) same locality as sample IEBR-24, but May 2011, Hoang Long; 1 female (IEBR-235) Me Linh station for biodiversity of IEBR, secondary forest, 4 November 2004, Huynh Thi Kim Hoi; Hai Phong: 5 males, 4 females (IEBR-202) Cat Ba NP, forest, on the way to the Frog Fond, $20^{\circ} 47^{\prime} 941 " \mathrm{~N}$ 106 59'580"E, 18 March 2012, Anh D. Nguyen; 1 male (IEBR-232) same locality as sample IEBR-202, but 25 April 2012, Nguyen Quang Truong; Quang Ninh: 2 males, 1 female (IEBR235) Bai Tu Long NP, Sau Nam Isl., forest, 22 February 2002, Anh D. Nguyen.

Remarks: The species seems to be widely distributed in northern Vietnam.

Distribution: Only known from northern Vietnam (Fig. 7).

\section{Nedyopus pictus (Brölemann, 1916)}

(Figs 5-6)

Vaulogerodesmus pictus Brölemann, 1916: 588, figures 1-2 [4]; Jeekel 1968: 61 [11]; Golovatch 1983: 182 [8]; Golovatch et al. 2002: 259-261, figures 1-9 [10]; Enghoff et al. 2004: 40 [6].

Nedyopus pictus:-Attems 1937: 140, figure 177 [2]; Chen et al. 2006: 4001 [5].

Diagnosis: The species can be distinguished from its congeners by smaller body size (less than $40 \mathrm{~mm}$ long and $5 \mathrm{~mm}$ wide); paraterga small, modestly developed, angular caudally, and not exceeding over posterior tergal contour; the fifth sternum with two independent, conical processes between coxae 4; gonopod femorite modestly expanded dorsally; solenophore with characteristically subsecuriform lamina medialis.

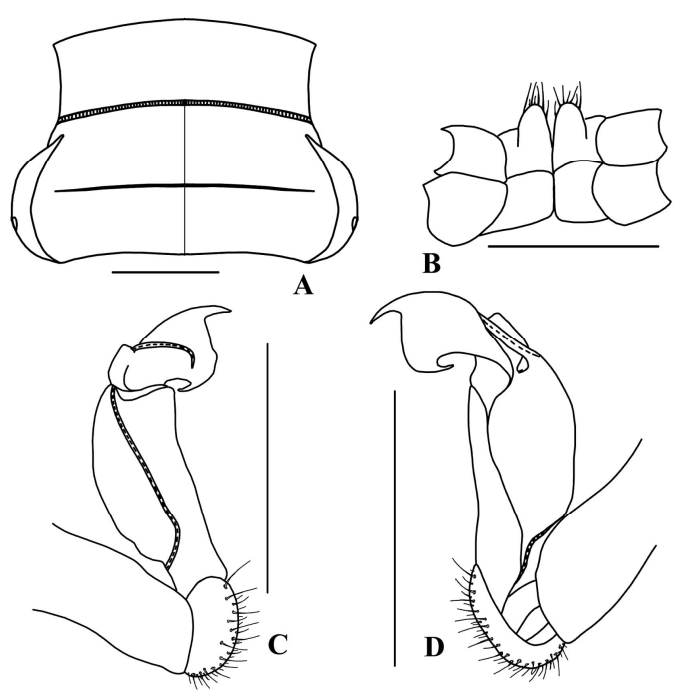

Figure 5. Nedyopus pictus (Brölemann, 1916), from Tam Dao National Park.

A. Body ring 10, dorsal view; B. sternum 5th, ventral view; C-D. right gonopod, lateral view and mesal view, respectively. Scale bar $=1 \mathrm{~mm}$.

Previous records: North of Vietnam (Brölemann 1916) [3], Vinh Phuc (Tam Dao NP) [10].

Material examined: Vinh Phuc: 2 males (IEBR-163) Tam Dao NP, around the town, 900-1,000 m a.s.l., April 2011, Hoang Long; 1 male (IEBR-170), same locality as sample IEBR-163, May 2011, Hoang Long.

Remarks: Brölemann (1916) [3] described the species from North of Vietnam, but did not state exact locality. The species was rediscovered in Tam Dao NP (Vinh Phuc Province) by Golovatch et al. (2002) [10]. The species has, so far, been only found in Tam Dao NP.

Distribution: Only known from northern Vietnam (Fig. 7). 


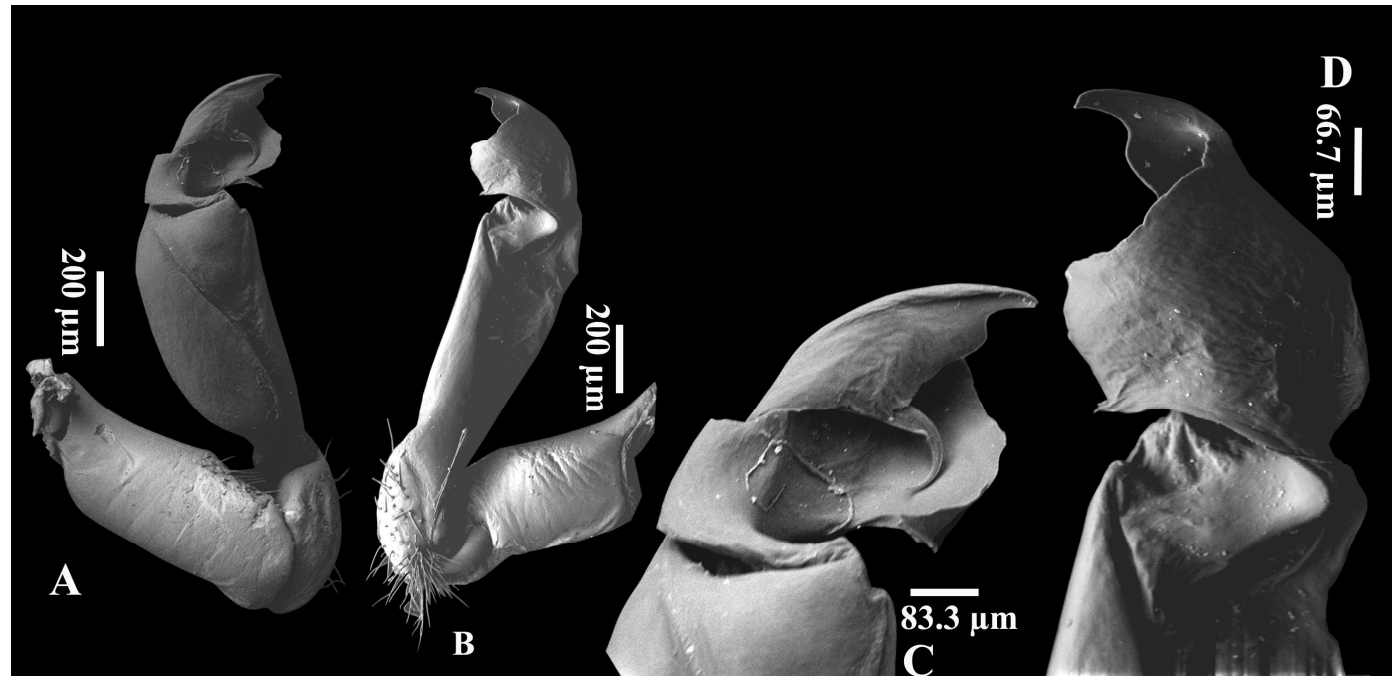

Figure 6. Nedyopus pictus (Brölemann, 1916), from Tam Dao National Park.

Left gonopod, lateral view (A), mesal view (B); tip of gonopod, lateral view (C), mesal view (D).

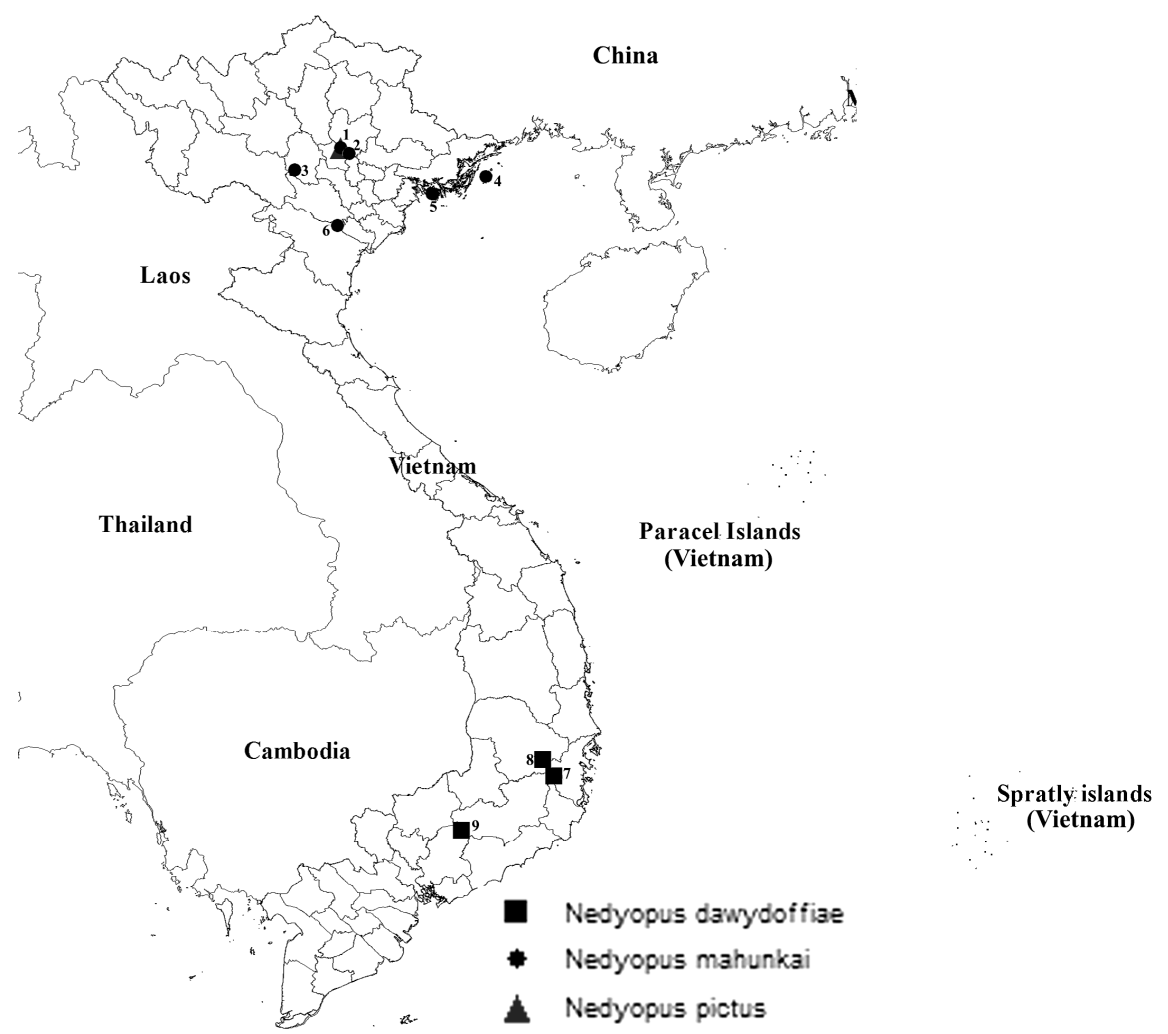

Figure 7. Distribution of Nedyopus species in Vietnam

1. Tam Dao NP (Vinh Phuc); 2. Me Linh (Vinh Phuc); 3. Xuan Son NP (Phu Tho); 4. Bai Tu Long NP (Quang Ninh); 5. Cat Ba NP (Hai Phong); 6. Cuc Phuong NP (Ninh Binh); 7. Bi Doup - Nui Ba NP (Lam Dong); 8. Chu Yan Sin NP (Dak Lak); 9. Cat Tien NP (Dong Nai). 
Acknowledgements: The author would like to express the deepest thanks to the VietnameseRusian Tropical Center, Bi Doup National Park, Tam Dao National Park, Cat Ba National Park for their supports to field expeditions. Special thanks are addressed to my colleagues for kindly providing material for this study. The paper is partly supported by the project No. IEBR.DT.02/13-14 of the Institute of Ecology and Biological Resources.

\section{REFERENCES}

1. Attems C., 1914. Die indo-australischen Myriopoden. Archiv für Naturgeschichte, 80A(4): 1-398.

2. Attems C., 1937. Myriapoda 3. Polydesmoidea I. Fam. Strongylosomidae. Das Tierreich, 68: 1-300.

3. Attems C., 1953. Myriopoden von Indochina. Expedition von Dr. C. Dawydoff (1938-1939). Mémoires du Muséum National d'Histoire Naturelle, \{N. S, Sér. A, Zool.\}, 5(3): 133-230.

4. Brölemann H. W., 1916. Essai de classification des Polydesmiens [Myriapodes]. Annales de la Société Entomologique de France, 84: 523-608.

5. Chen C.-C., Golovatch S. I., Chang H.-W., 2006. The millipede tribe Nedyopodini, with special reference to the fauna of Taiwan (Diplopoda: Polydesmida: Paradoxosomatidae). Journal of Natural History, 39(47): 3997-4030.

6. Enghoff H., Golovatch S. I., Nguyen D. A., 2004. A review of the millipede fauna of Vietnam (Diplopoda). Arthropoda Selecta, 13(1/2): 29-43.

7. Golovatch S. I., Semenyuk I. I., 2010. On several new or poorly known Oriental Paradoxosomatidae (Diplopoda, Polydesmida), X. Arthropoda Selecta, 19(3): 123-127.

8. Golovatch S. I., 1983. Millipedes (Diplopoda) of the fauna of Vietnam. In: Medvedev L. N. (Eds), Fauna and ecology of the animals of Vietnam. Nauka publisher, Moscow. 207pp.

9. Golovatch S. I., 2009. On several new or poorly known Oriental Paradoxosomatidaen (Diplopoda, Polydesmida), VIII. Arthropoda Selecta, 18(1/2): 1-7.

10. Golovatch S. I., Wytwer J., Jędryczkowski W. B., 2002. The millipede Vaulogerodesmus pictus (Brölemann, 1916) rediscovered, with a review of this Southeast Asian genus (Diplopoda: Polydesmida: Paradoxosomatidae). Arthropoda Selecta, 11(4): 259-264.

11. Jeekel C. A. W., 1968. On the classification and geographical distribution of the family Paradoxosomatidae (Diplopoda, Polydesmida). Bronder-Offset Rotterdam, privately published: 1-162.

12. Korsós Z., Golovatch S. I., 1989. Addenda to the millipede fauna of Vietnam (Diplopoda). Acta Zoologica Hungarica, 35(3/4): 211-220.

13. Verhoeff K. W., 1940. Zur Kenntnis ostasiatischer Diplopoden. V (Polydesmoidea). Zoologischer Anzeiger, 131(5/6): 129-145.

14. Wang Y. M., 1957. Serica 1g: Records of myriapods on Taiwan Islands (4) Six New Polydesmids. Quarterly Journal of the Taiwan Museum, 10(3/4): 103-111. 


\section{GIỐNG CHÂN KÉP NEDYOPUS ATTEMS, 1914 (DIPLOPODA: POLYDESMIDA: PARADOXOSOMATIDAE) Ở VIẸT NAM}

\section{Nguyễn Đức Anh}

Viện Sinh thái và Tài nguyên sinh vật, Viện Hàn lâm KH \& CN Việt Nam

\section{TÓM TẮT}

Bài báo trình bày đặc điểm chẩn loại giống và các đặc điểm chẩn loại của 3 loài chân kép thuộc giống Nedyopus Attems, 1914 ở Việt Nam: N. dawydoffiae (Attems, 1953), N. mahunkai (Korsós \& Golovatch, 1989) và N. pictus (Brölemann, 1916). Phân bố của các loài và khoá định loại 3 loài ở Việt Nam cũng được trình bày trong bài báo.

Khóa định loại các loài chân kép thuộc giống Nedyopus ở Việt Nam

1. Chiều dài cơ thể khoảng $40 \mathrm{~mm}$. Tấm bụng thứ 5 có một phiến mỏng, lớn giữa đốt háng thứ 4 (Hình 1B). Đốt đùi chân giao phối có 2 cạnh song song, nhưng hơi mở rộng về phía cuối (Hình 1C-D, 2) .N. dawydoffiae

- Chiều dài cơ thể khoảng $32 \mathrm{~mm}$. Tấm bụng thứ 5 có hai mấu hình nón, tròn giữa đốt háng thứ 4 . Đốt đùi chân giao phối mở rộng nhiều về phía cuối, hai cạnh không song song.

2. Tấm bên lưng phát triển bình thường, luôn luôn có góc phía cuối; không phát triển qua cạnh sau của tấm lưng (Hình $5 \mathrm{~A}$ ). Tấm bụng thứ 5 có hai mấu hình nón, có tơ và tách rời nhau giữa đốt háng thứ 4 (Hình 5B). Lamina medialis của solenophore chân giao phối có hình rìu đặc trưng (Hình 5C-D, 6)....... pictus

- Tấm lưng bên rất phát triển, gần như nhọn ở phía cuối; đôi khi phát triển vượt qua cạnh sau của tấm lưng (Hình $3 \mathrm{~A}$ ). Tấm bụng thứ 5 có hai mấu hình nón, có tơ và gần nhau giữa đốt háng thứ 4 (Hình $3 \mathrm{~B}$ ). Lamina medialis của solenophore chân giao phối không có hình rìu (Hình 3C-D, 4) .N. mahunkai

Từ khóa: Paradoxosomatidae, Nedyopus, Chân kép, phân bố, Việt Nam.

Ngày nhận bài: 8-8-2013 